\title{
ENERGY-MOMENTUM SCHEME WITH DRILLING DEGREES OF FREEDOM FOR COMPOSITES WITH CURVATURE-TWIST STIFFNESS
}

\author{
MICHAEL GROSS ${ }^{1}$, JULIAN DIETZSCH ${ }^{2}$ AND INIYAN KALAIMANI ${ }^{3}$ \\ ${ }^{1}$ Technische Universität Chemnitz, Reichenhainer Straße 70, D-09126 Chemnitz, \\ https://www.tu-chemnitz.de/mb/TMD/, michael.gross@mb.tu-chemnitz.de \\ 2 julian.dietzsch@mb.tu-chemnitz.de \\ 3 iniyan.kalaimani@mb.tu-chemnitz.de
}

Key words: Fiber-reinforced Material, Generalized Continuum, Bespoke Finite Element Method.

\begin{abstract}
This paper is motivated by the increasing application of 3D fiber-reinforced composites in rotating systems [1]. In 2D fiber-reinforced composites, single fibers with a diameter in the range of micrometers are embedded in a matrix material. But, these composites are prone to delamination damage, wherefore the development of 3D composites has been undertaken. Here, fiber bundles are woven, knitted, braided or stitched, in order to fix the fibers before they are surrounded by a matrix material. From a material modelling point of view, these two kind of composites make a huge difference, because a fiber bundle has to be considered as a beam-like structure with curvature-twist (bending as well as twisting) stiffness, in addition to the usual stretching stiffness (cf. [2]). The former is then responsible for the increasing strength-to-weight ratio of 3D fiber-reinforced composites for thin-walled lightweight structures. Therefore, 3D fiber-reinforced composites demand for a bespoke simulation technique.

We have to consider a representative volume element, in which secondary effects as a micro inertia and a curvature-twist stiffness must be taken into account. We introduce these secondary effects in a continuum formulation by means of independent drilling degrees of freedom (cf. [3]). The resulting nonisothermal constrained micropolar continuum is derived by a mixed principle of virtual power (cf. [4]). This variational principle simultaneously generates in the discrete setting a mixed B-bar method and a Galerkin-based energy-momentum scheme of higher order. We also take into account viscoelastic material behaviour, which arises from a mixture of organic and inorganic fibers in a polymeric matrix material. Representative numerical examples demonstrate the twisting and bending stiffness of the fiber bundles.
\end{abstract}

\section{INTRODUCTION}

Since we aim at a finite element method, our strategy starts with consideration of generalized continuum mechanics and unsymmetric stress tensors. The reason is that anisotropic Cauchy continua with a symmetric stress tensor, formulated by idempotent structural tensors $\boldsymbol{A}_{0}=\boldsymbol{a}_{0} \otimes \boldsymbol{a}_{0}$ with $\left\|\boldsymbol{a}_{0}\right\|=1$, where the vector $\boldsymbol{a}_{0}$ denotes the fiber direction vector in the initial configuration $\mathscr{B}_{0}$ and $\otimes$ the dyadic product of tensors, assume single fibers without a curvature-twist stiffness. But, bending tests with a fiber roving 

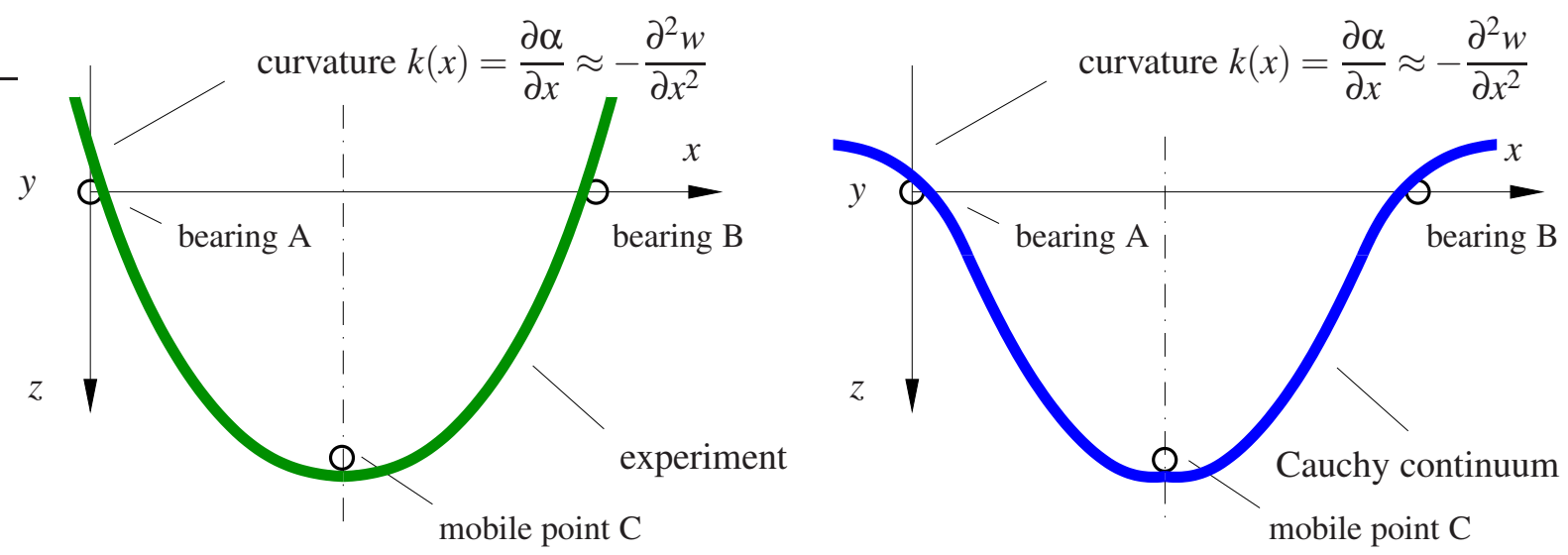

Figure 1: Three point bending test: experiment versus Cauchy theory (see Reference [5]). The introduction of drilling degrees of freedom $\alpha$ can be motivated by the bending of a two-dimensional Euler-Bernoulli beam in applied mechanics. The curvature $k(x)$ of the beam axis can be formulated very easy by a drilling degree of freedom $\alpha$ with respect to the $y$-axis, instead of using the displacement $w$ in $z$-direction.

composite reveal, that the roving bending stiffness has a large influence on the deformation. Beam ends are stiff and beam curvatures are large, in contrast to finite element simulations with a Cauchy continuum and structural tensors (see Figure 1). Such curvature effects can be modelled by tensor invariants associated with $(i)$ a second-order material gradient $\operatorname{Grad}[\boldsymbol{F}]:=\operatorname{Grad}\left[\operatorname{Grad}\left[\boldsymbol{\varphi}_{t}\right]\right]$ with respect to a point $\boldsymbol{X} \in \mathscr{B}_{0}$ of the deformation mapping $\varphi_{t}: \mathscr{B}_{0} \rightarrow \mathscr{B}_{t}$ to the current configuration $\mathscr{B}_{t}$ or (ii) a material rotation

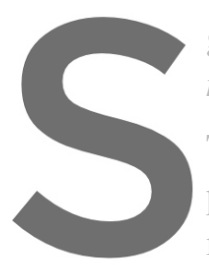
gradient $G:=\operatorname{Grad}[\alpha]$ $n_{\mathrm{dim}}$-dimensional space. Therefore, beside the deformation mapping $\varphi_{t}$, we pendent fields. As we micropolar continuum. Nore precisely, we introd
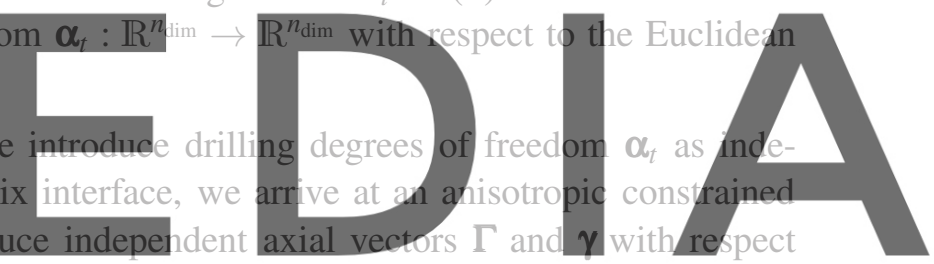

to the initial configuration $\mathscr{B}_{0}$ and the current configuration $\mathscr{B}_{t}$, respectively. As curvature measure,

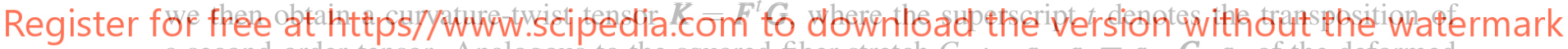
a second-order tensor. Analogous to the squared fiber stretch $C_{F}:=a_{t} \cdot a_{t} \equiv a_{0} \cdot C \cdot a_{0}$ of the deformed fiber direction vector $a_{t}$, being also the trace of the product tensor $C_{F}:=A_{0} C$ of structural tensor $A_{0}$ and right Cauchy-Green tensor $\boldsymbol{C}:=\boldsymbol{F}^{t} \boldsymbol{F}$, we show that the fiber twist $T_{F}:=\boldsymbol{a}_{t} \cdot \operatorname{grad}[\boldsymbol{\alpha}] \cdot \boldsymbol{a}_{\boldsymbol{t}} \equiv \boldsymbol{a}_{0} \cdot \boldsymbol{K} \cdot \boldsymbol{a}_{0}$ per unit length is the trace of the product tensor $\boldsymbol{K}_{F}:=\boldsymbol{A}_{0} \boldsymbol{K}$ of the structural tensor and the curvature-twist tensor $\boldsymbol{K}$. Here, we denote by $\operatorname{grad}[\bullet]$ the spatial gradient with respect to a point $\boldsymbol{x} \in \mathscr{B}_{t}$. Second, we show that the fiber bending per unit length is described by $-2 I_{2}^{\boldsymbol{K}_{F}}:=B_{F}-T_{F}^{2}$, with $B_{F}:=\boldsymbol{a}_{0} \cdot \boldsymbol{K} \boldsymbol{K}^{t} \cdot \boldsymbol{a}_{0}$, where $I_{2}^{K_{F}}$ denotes the second principal tensor invariant of the fiber curvature-twist tensor $\boldsymbol{K}_{F}$.

Finally, we derive each weak form by using the principle of virtual power, instead of using the principle of virtual work or least action. The reason can be explained by the Simo-Taylor-Pister functional

$$
\Pi_{\mathrm{STP}}(\boldsymbol{\varphi}, \tilde{J}, \tilde{p}):=\int_{\mathscr{B}_{0}} \Psi^{\mathrm{iso}}(\boldsymbol{\varphi}) \mathrm{d} V+\int_{\mathscr{B}_{0}} \Psi^{\mathrm{vol}}(\tilde{J}) \mathrm{d} V-\int_{\mathscr{B}_{0}}(\tilde{J}-\operatorname{det}[\operatorname{Grad}[\boldsymbol{\varphi}]]) \tilde{p} \mathrm{~d} V
$$

which introduces an independent volume dilatation $\tilde{J}$ and pressure $\tilde{p}$. The operator $\operatorname{det}[\bullet]$ denotes the determinant of a second-order tensor, whereby the volume densities $\Psi^{\text {iso }}$ and $\Psi^{\mathrm{vol}}$ designate an isochoric and volumetric free energy function, respectively. Both, the principles of virtual work and of least action 
derive a weak form for the volume dilatation on the variable level, given by

$$
\int_{\mathscr{B}_{0}} G(\boldsymbol{\varphi}, \tilde{J}) \delta \tilde{p} \mathrm{~d} V=0 \quad \text { with } \quad G(\boldsymbol{\varphi}, \tilde{J}):=\tilde{J}-\operatorname{det}[\operatorname{Grad}[\boldsymbol{\varphi}]]
$$

But, in the energy-momentum time integration, the preservation of the balance law of total energy

$$
\int_{\mathscr{B}_{0}} H_{t_{n+1}} \mathrm{~d} V-\int_{\mathscr{B}_{0}} H_{t_{n}} \mathrm{~d} V=\int_{t_{n}}^{t_{n+1}} \int_{\mathscr{B}_{0}}\left[\frac{\partial H}{\partial \dot{\varphi}} \cdot \ddot{\varphi}+\frac{\partial H}{\partial \varphi} \cdot \dot{\varphi}+\frac{\partial H}{\partial \tilde{J}} \dot{\tilde{J}}\right] \mathrm{d} V \mathrm{~d} t
$$

of one step $\left[t_{n}, t_{n+1}\right]$ on the time axis $t$, with the energy density

$$
H:=\frac{1}{2} \rho_{0} \dot{\boldsymbol{\varphi}} \cdot \dot{\boldsymbol{\varphi}}+\Psi^{\mathrm{iso}}(\boldsymbol{\varphi})+\Psi^{\mathrm{vol}}(\tilde{J})
$$

and $\rho_{0}$ as material volume density in the inital configuration, requires a weak form

$$
\int_{t_{n}}^{t_{n+1}} \int_{\mathscr{B}_{0}} \dot{G}(\dot{\varphi}, \dot{\tilde{J}}) \delta \tilde{p} \mathrm{~d} V \mathrm{~d} t=0
$$

for the time derivative $\dot{\tilde{J}}$ of the independent volume dilatation $\tilde{J}$. Such a weak form can be directly obtained by a variational principle

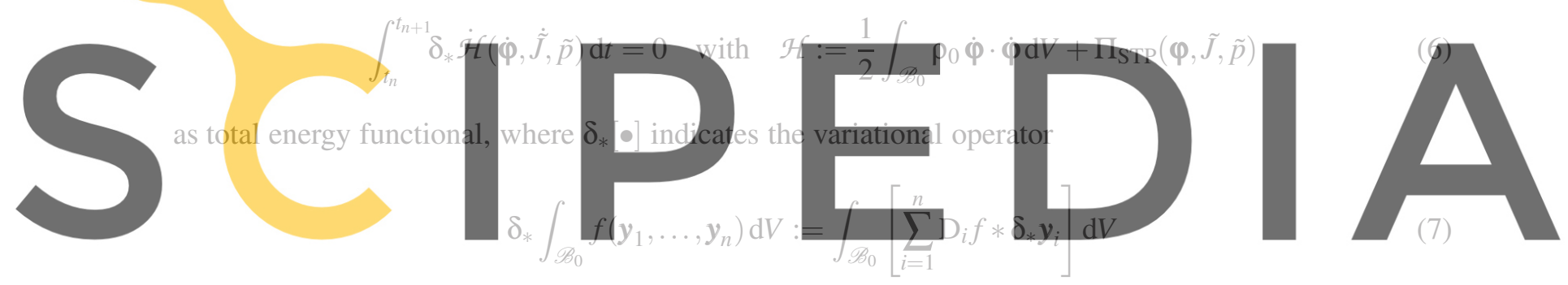

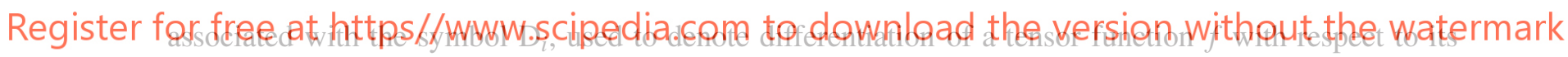

$i$-th argument tensor. Here, the symbol $*$ indicates a corresponding tensor contraction. We also use the symbol $\mathrm{D}$ to denote differentiation if $f$ has onity one argument.

\section{CONSTITUTIVE MODEL}

We begin by deriving the fiber twisting kinematics and the corresponding strain energy. The twist of a fiber can be easily derived by means of a projection of the drilling degrees of freedom $\boldsymbol{\alpha}$ on the current fiber direction $\boldsymbol{a}_{t}$ (see Figure 2). The twist of a fiber relative to a point $P$ in the current configuration $\mathscr{B}_{t}$ with unit length $\mathrm{d} \xi$ is then the projection

$$
\mathrm{d} \boldsymbol{\alpha} \cdot \boldsymbol{a}_{t}=(\operatorname{grad}[\boldsymbol{\alpha}] \cdot \mathrm{d} \boldsymbol{x}) \cdot \boldsymbol{a}_{t}=\left(\operatorname{grad}[\boldsymbol{\alpha}] \cdot \boldsymbol{a}_{t} \mathrm{~d} \xi\right) \cdot \boldsymbol{a}_{t}
$$

of the differential $\mathrm{d} \boldsymbol{\alpha}$ of the drilling degrees of freedom. The twist per unit length is the quadratic form

$$
T_{F}:=\boldsymbol{a}_{t} \cdot \operatorname{grad}[\boldsymbol{\alpha}] \cdot \boldsymbol{a}_{t}=\boldsymbol{K}: \boldsymbol{A}_{0}=\boldsymbol{K}_{F}: \boldsymbol{I}
$$




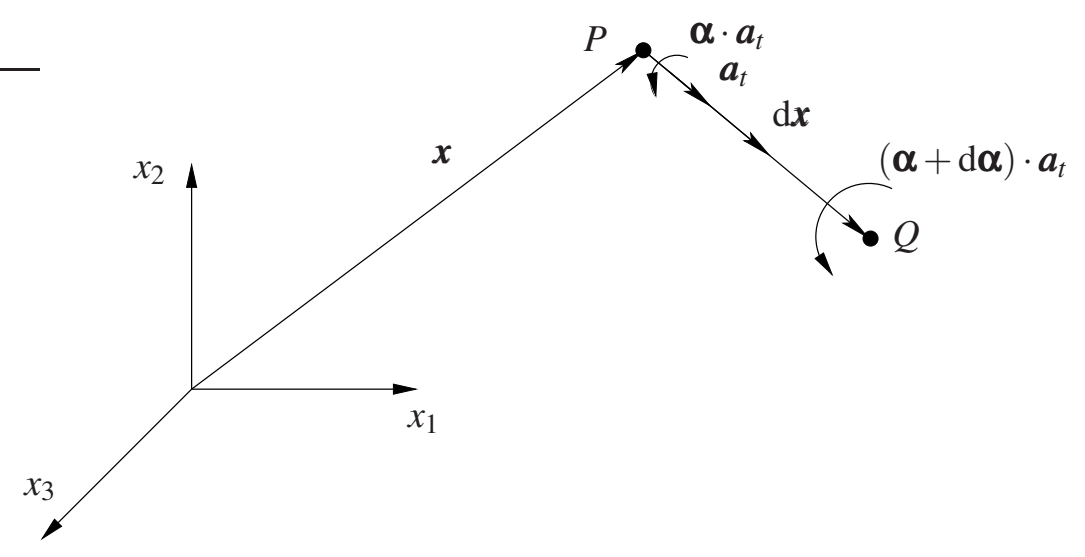

Figure 2: Twist of a fiber bundle based on drilling degrees of freedom (cf. Reference [6]).

with respect to the spatial rotation gradient $\operatorname{grad}[\boldsymbol{\alpha}]$, or the trace of the fiber curvature-twist tensor $\boldsymbol{K}_{F}$, respectively. The second-order tensor $\boldsymbol{I}$ indicates the identity tensor. In this paper, we restrict us to a quadratic fiber curvature-twist strain energy

$$
\Psi_{F_{\mathrm{SVK}}}^{\mathrm{ctw}}:=\Psi_{F}^{\mathrm{twi}}+\Psi_{F}^{\mathrm{cur}}=\frac{1}{2} K_{\mathrm{twi}}\left(T_{F}\right)^{2}+\frac{1}{2} \mu_{\mathrm{cur}}\left(b_{F}\right)^{2}
$$

with a fiber twisting function $\Psi_{F}^{\text {twi }}$ associated with a fiber twisting stiffness parameter $K_{\mathrm{twi}}$, and a fiber bending function $\Psi_{F}^{\text {cur }}$ related with a fiber bending
length, $b_{F}$, can be related with a deviatoric part of
the following by elaborating in three steps that the
strain energy function with respect to the floer curva
First, we recall the structure of the St. Venant-Kit
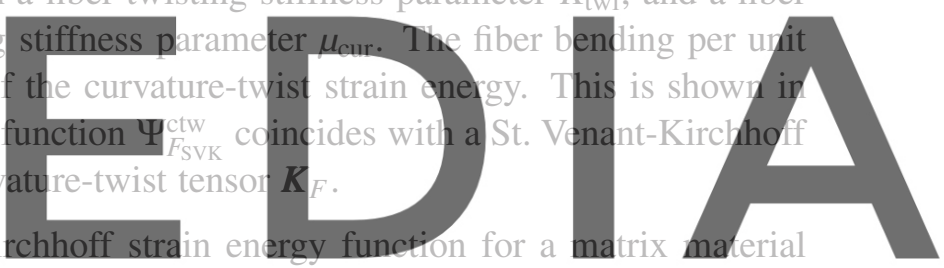

based on a spherical and a deviatoric contribution, given by

Register for free at https//www.scipedia.com to download the version without the watermark

$$
\Psi_{\mathrm{SVK}}^{\mathrm{EV}}:=\Psi_{\mathrm{SVK}}^{\mathrm{vvl}}+\Psi_{\mathrm{SVK}}^{\mathrm{deV}}=\frac{1}{2} K\left(E^{\mathrm{Vol}}\right)^{2}+\frac{1}{2} \mu\left(E^{\mathrm{deV}}\right)^{2}=\frac{1}{2}[E: I]^{2}+\mu E: E
$$

with respect to the Green-Lagrange strain tensor $\boldsymbol{E}:=[\boldsymbol{C}-\boldsymbol{I}] / 2$. The material parameter $K$ denotes the bulk modulus and $\mu$ the shear modulus. The spherical strain

$$
E^{\mathrm{vol}}:=\sqrt{n_{\mathrm{dim}} \operatorname{sph}_{\boldsymbol{I}}[\boldsymbol{E}]: \operatorname{sph}_{\boldsymbol{I}}[\boldsymbol{E}]}=\boldsymbol{E}: \boldsymbol{I}
$$

leads to the volumetric strain energy $\Psi_{\mathrm{SVK}}^{\mathrm{vol}}$, and the conjugated deviatoric strain

$$
E^{\mathrm{dev}}:=2 \sqrt{\frac{1}{2} \operatorname{dev}_{\boldsymbol{I}}[\boldsymbol{E}]: \operatorname{dev}_{\boldsymbol{I}}[\boldsymbol{E}]}=\sqrt{2 \operatorname{dev}_{\boldsymbol{I}}[\boldsymbol{E}]: \operatorname{dev}_{\boldsymbol{I}}[\boldsymbol{E}]}=\sqrt{2\left[\boldsymbol{E}: \boldsymbol{E}-\frac{1}{n_{\mathrm{dim}}}\left(E^{\mathrm{vol}}\right)^{2}\right]}
$$

generates the energy due to matrix distorsions. The operator $\operatorname{sph}_{\boldsymbol{D}}[(\bullet)]:=1 /(\boldsymbol{D}: \boldsymbol{I})[(\bullet): \boldsymbol{I}] \boldsymbol{D}$ defines the spherical tensor part with respect to any second-order tensor $\boldsymbol{D}$, and $\operatorname{dev}_{\boldsymbol{D}}[(\bullet)]:=(\bullet)-\operatorname{sph}_{\boldsymbol{D}}[(\bullet)]$ the conjugated traceless deviatoric tensor. In the end, we obtain in Eq. (11) the well-known St. VenantKirchhoff strain energy function with respect to Lamé's first and second parameter $\mu$ and $\lambda:=K-$ $2 \mu / n_{\text {dim }}$, respectively. 
This concept can be also applied in order to design a St. Venant-Kirchhoff fiber strain energy

$$
\Psi_{F_{\mathrm{SVK}}}^{\mathrm{ela}}:=\Psi_{F}^{\mathrm{str}}+\Psi_{F}^{\mathrm{dis}}=\frac{1}{2} K_{F}\left(E_{F}^{\mathrm{str}}\right)^{2}+\frac{1}{2} \mu_{F}\left(E_{F}^{\mathrm{dis}}\right)^{2}=\frac{\lambda_{F}}{2}\left[\boldsymbol{E}_{F}: \boldsymbol{I}\right]^{2}+\mu_{F} \boldsymbol{E}_{F}: \boldsymbol{E}_{F}
$$

with the Lamé parameter $\lambda_{F}:=K_{F}-2 \mu_{F}$. Now, the spherical contribution of the fiber strain tensor $\boldsymbol{E}_{F}:=\boldsymbol{A}_{0} \boldsymbol{E}$ with respect to the structural tensor $\boldsymbol{A}_{0}$ introduces the fiber stretch

$$
E_{F}^{\mathrm{str}}:=\sqrt{\operatorname{sph}_{\boldsymbol{A}_{0}}\left[\boldsymbol{E}_{F}\right]: \operatorname{sph}_{\boldsymbol{A}_{0}}\left[\boldsymbol{E}_{F}\right]}=\boldsymbol{E}: \boldsymbol{A}_{0}=\boldsymbol{E}_{F}: \boldsymbol{I}
$$

of the one-dimensional fiber. The deviatoric strain

$$
E_{F}^{\mathrm{dis}}:=\sqrt{2 \operatorname{dev}_{\boldsymbol{A}_{0}}\left[\boldsymbol{E}_{F}\right]: \operatorname{dev}_{\boldsymbol{A}_{0}}\left[\boldsymbol{E}_{F}\right]}=\sqrt{2\left[J_{5}^{\mathrm{E}}-\left(E_{F}^{\mathrm{str}}\right)^{2}\right]}
$$

includes the basic invariant $J_{5}^{\mathrm{E}}:=\boldsymbol{a}_{0} \cdot \boldsymbol{E} \boldsymbol{E} \cdot \boldsymbol{a}_{0}$ of the symmetric Green-Lagrange strain tensor. This contribution leads to a stress component arising from distorsions relative to the fiber direction $a_{0}$. We highlight that the spherical and deviatoric operator for the fiber is defined with respect to the tensor $A_{0}$. By using this concept, we obtain a St. Venant-Kirchhoff fiber curvature-twist strain energy

$$
\Psi_{F_{\mathrm{SVK}}}^{\mathrm{ctw}}:=\Psi_{F}^{\mathrm{twi}}+\Psi_{F}^{\mathrm{cur}}=\frac{1}{2} K_{\mathrm{twi}}\left(T_{F}\right)^{2}+\frac{1}{2} \mu_{\mathrm{cur}}\left(b_{F}\right)^{2}=\frac{\lambda_{\mathrm{ctw}}}{2}\left[\boldsymbol{K}_{F}: \boldsymbol{I}\right]^{2}+\mu_{\mathrm{cur}} \boldsymbol{K}_{F}: \boldsymbol{K}_{F}
$$

with the Lamé parameter $\lambda_{\mathrm{ctw}}:=K_{\mathrm{twi}}-2 \mu_{\mathrm{cur}}$. The spherical part describes the twisting energy $\Psi_{F}^{\mathrm{twi}}$ as shown in Eqs. (9), and (10),
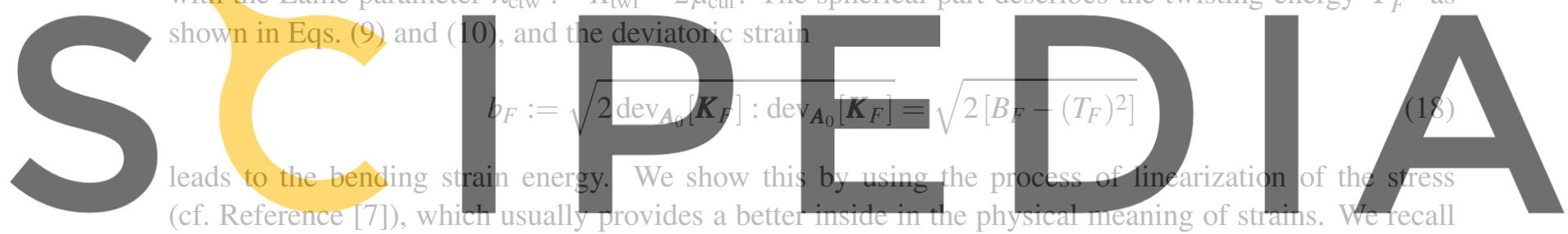

this process by means of the first Piola-Kirchhoff stress tensor

Register for free at https//www.scipedia.com to download the version without the watermark

$$
P_{\mathrm{SVK}}^{\mathrm{ela}}(F):=F S_{\mathrm{SVK}}^{\mathrm{ela}}(E(F))=F \frac{\partial \Psi_{\mathrm{SVK}}^{\mathrm{ela}}(\mathbb{E}(\boldsymbol{F}))}{\partial E}=F\{\lambda[E(F): I] I+2 \mu E(F)\}
$$

of the St. Venant-Kirchhoff strain energy function of a matrix material. Here, we designate by $\boldsymbol{S}_{\mathrm{SVK}}^{\mathrm{ela}}$ the second Piola-Kirchhoff stress tensor of the matrix material. The linearization with respect to the deformation gradient $\boldsymbol{F}_{0}=\boldsymbol{I}$ in the reference configuration $\mathscr{B}_{0}$ leads to the Cauchy stress of Hooke's law

$$
\frac{\mathrm{d}}{\mathrm{d} s}\left[\boldsymbol{P}_{\mathrm{SVK}}^{\mathrm{ela}}(\boldsymbol{I}+s \Delta \boldsymbol{H})\right]_{s=0}=\lambda[\boldsymbol{\varepsilon}: \boldsymbol{I}] \boldsymbol{I}+2 \mu \boldsymbol{\varepsilon}=: \boldsymbol{\sigma}_{\mathrm{H}}^{\text {ela }}=\frac{\partial \Psi_{\mathrm{H}}^{\text {ela }}}{\partial \boldsymbol{\varepsilon}}
$$

where $\boldsymbol{\varepsilon}:=\left[\Delta \boldsymbol{H}+\Delta \boldsymbol{H}^{t}\right] / 2$ designates the linearized strain tensor. The tensor $\Delta \boldsymbol{H}:=\partial \Delta \boldsymbol{x} / \partial \boldsymbol{X}$ represents the linearized displacement gradient. The strain energy function $\Psi_{\mathrm{H}}^{\text {ela }}$ of Hooke's law then takes the form

$$
\Psi_{\mathrm{H}}^{\mathrm{ela}}=\frac{\lambda}{2}[\boldsymbol{\varepsilon}: \boldsymbol{I}]^{2}+\mu \boldsymbol{\varepsilon}: \boldsymbol{\varepsilon}=\frac{1}{2} K\left(e^{\mathrm{vol}}\right)^{2}+\frac{1}{2} \mu\left(e^{\mathrm{dev}}\right)^{2}
$$

The trace $\boldsymbol{\varepsilon}: \boldsymbol{I}$ of the linearized strain tensor can be then identified via the divergence $\operatorname{div}[\Delta \boldsymbol{x}]$ of the displacement field $\Delta x$ as the volume dilatation $\Delta V / V_{0}$ of the deformed configuration with respect to $\mathscr{B}_{0}$. 
We now apply this linearization procedure on the Piola-Kirchhoff couple stress tensor, given by

$$
\begin{aligned}
& \boldsymbol{P}_{K}^{\mathrm{SVK}}(\boldsymbol{F}, \boldsymbol{G}):=\boldsymbol{F} \boldsymbol{S}_{K}^{\mathrm{SVK}}(\boldsymbol{F}, \boldsymbol{G}) \equiv \boldsymbol{F} \frac{\partial \Psi_{F_{\mathrm{SVK}}}^{\mathrm{ctw}}(\boldsymbol{K}(\boldsymbol{F}, \boldsymbol{G}))}{\partial \boldsymbol{K}} \\
& \boldsymbol{P}_{K}^{\mathrm{SVK}}(\boldsymbol{F}, \boldsymbol{G})=\boldsymbol{F}\left\{\lambda_{\mathrm{ctw}}\left[\boldsymbol{K}_{F}(\boldsymbol{F}, \boldsymbol{G}): \boldsymbol{I}\right] \boldsymbol{A}_{0}+2 \mu_{\mathrm{cur}} \boldsymbol{K}_{F}(\boldsymbol{F}, \boldsymbol{G})\right\}
\end{aligned}
$$

but we linearize with respect to the deformation gradient $\boldsymbol{F}_{0}=\boldsymbol{I}$ as well as the material rotation gradient $\boldsymbol{G}_{0}=\boldsymbol{O}$ in the reference configuration $\mathscr{B}_{0}$. Here, the second-order tensor $\boldsymbol{O}$ denotes the zero tensor. In this way, we arrive at the Kirchhoff couple stress

$$
\frac{\mathrm{d}}{\mathrm{d} s}\left[\boldsymbol{P}_{K}^{\mathrm{SVK}}(\boldsymbol{I}+s \Delta \boldsymbol{H}, \boldsymbol{O}+s \Delta \boldsymbol{G})\right]_{s=0}=\boldsymbol{\lambda}_{\mathrm{ctw}}\left[\boldsymbol{\kappa}_{F}: \boldsymbol{I}\right] \boldsymbol{A}_{0}+2 \mu_{\mathrm{cur}} \boldsymbol{\kappa}_{F}=: \boldsymbol{\mu}_{K}^{\mathrm{H}}=\frac{\partial \Psi_{K}^{H}}{\partial \boldsymbol{\kappa}_{F}} \frac{\partial \mathbf{\kappa}_{F}}{\partial \Delta \boldsymbol{G}}
$$

of Hooke's couple law for the fiber bundles, formulated by the linearized fiber curvature-twist tensor

$$
\frac{\mathrm{d}}{\mathrm{d} s}\left[\boldsymbol{K}_{F}(\mathbb{I}+s \Delta H, O+s \Delta G)\right]_{s=0}=A_{0} \Delta G=: \mathfrak{K}_{F}
$$

The corresponding Hooke's couple strain energy function $\Psi_{K}^{H}$ can be written by using Lamé parameters or a spherical and a deviatoric part, respectively, as
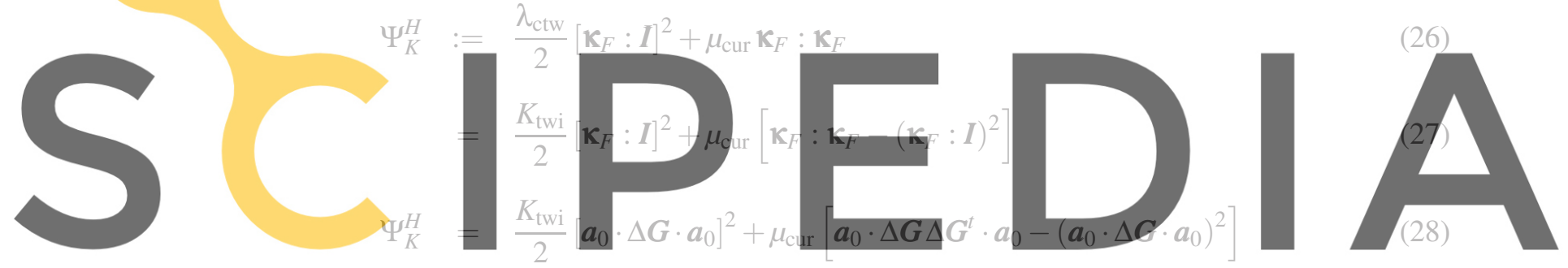

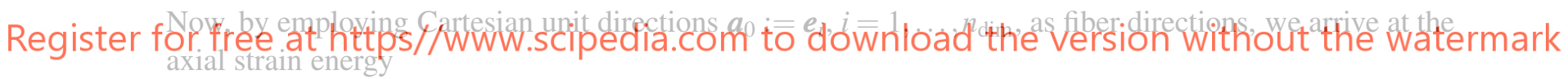

$$
\left.\Psi_{K}^{H}\right|_{\boldsymbol{a}_{0}:=\boldsymbol{e}_{i}}=\frac{K_{\mathrm{twi}}}{2}\left[\frac{\partial \Delta \alpha_{i}}{\partial X_{i}}\right]^{2}+\mu_{\mathrm{cur}} \sum_{\substack{j=1 \\ i \neq j}}^{n_{\mathrm{dim}}}\left[\frac{\partial \Delta \alpha_{i}}{\partial X_{j}}\right]^{2}
$$

with respect to the $i$-th Cartesian unit direction. We recognize in the spherical part (first term) the twisting modes, and in the remainder deviatoric strain energy the bending modes of the linearized rotation gradient

$$
\Delta \boldsymbol{G}:=\frac{\partial \Delta \alpha_{i}}{\partial X_{j}} \boldsymbol{e}_{i} \otimes \boldsymbol{e}_{j}
$$

with respect to Cartesian unit directions. Consequently, a curvature-twist law for the fiber bundles reads

$$
\Psi_{K}(\boldsymbol{K}) \doteq \Psi_{F_{\mathrm{SvK}}}^{\mathrm{ctw}}\left(\boldsymbol{K} ; \boldsymbol{A}_{0}\right):=\frac{\mu_{K}\left(l_{t}\right)^{2}}{2}\left(T_{F}\right)^{2}+\mu_{K}\left(l_{b}\right)^{2} B_{F}=\frac{\mu_{K}\left(l_{t}\right)^{2}}{2}\left(\boldsymbol{K}_{F}: \boldsymbol{I}\right)^{2}+\mu_{K}\left(l_{b}\right)^{2} \boldsymbol{K}_{F}: \boldsymbol{K}_{F}
$$

This St. Venant-Kirchhoff strain energy is our starting point of a development of non-linear curvaturetwist strain energy functions. In the development of energy-momentum schemes, a St. Venant-Kirchhoff 
strain energy function is also recommended as starting point (see Reference [8]). Motivated by Reference [9], we introduce a curvature-twist stiffness parameter $\mu_{K}$ associated with length scales $l_{t}$ and $l_{b}$ for twisting and bending, respectively, of the fiber bundles.

As anisotropic continuum force model, we consider a decoupled thermo-viscoelastic free energy for matrix and fiber material. In the decoupled elastic strain energy

$$
\Psi^{\text {ela }}\left(\boldsymbol{C}, \tilde{C}_{V}, \tilde{C}_{F} ; \boldsymbol{A}_{0}\right)=\Psi_{M}^{\text {iso }}\left(I_{1}^{\mathrm{C}}, I_{2}^{\mathrm{C}}, I_{3}^{\mathrm{C}}, I_{4}^{\mathrm{C}}\right)+\Psi_{M}^{\mathrm{vol}}\left(\tilde{C}_{V}\right)+\Psi_{F}^{\text {ela }}\left(\tilde{C}_{F}\right)
$$

we consider the volumetric strain invariant $C_{\mathrm{V}}:=(\operatorname{det}[\boldsymbol{C}])^{1 / n_{\mathrm{dim}}}$ and the squared fiber stretch $C_{F}:=\boldsymbol{C}: \boldsymbol{A}_{0}$ as independent fields $\tilde{C}_{V}$ and $\tilde{C}_{F}$, respectively. Note that we denote, in this paper, by

$$
I_{1}^{\mathrm{C}}:=\boldsymbol{C}: \boldsymbol{I} \quad I_{2}^{\mathrm{C}}:=\frac{1}{2}\left[\left(I_{1}^{\mathrm{C}}\right)^{2}-\boldsymbol{C}^{2}: \boldsymbol{I}\right] \quad I_{3}^{\mathrm{C}}:=\operatorname{det}[\boldsymbol{C}] \quad I_{4}^{\mathrm{C}}:=\boldsymbol{C}: \boldsymbol{A}_{0} \equiv \boldsymbol{a}_{t} \cdot \boldsymbol{a}_{t}=\left\|\boldsymbol{a}_{t}\right\|^{2}
$$

the principal tensor invariants of the right Cauchy-Green tensor $\boldsymbol{C}$ and the structural tensor $\boldsymbol{A}_{0}$. As we consider a fiber-reinforced continuum body $\mathscr{B}$, moving in the Euclidean space $\mathbb{R}^{n_{\mathrm{dim}}}$ with constant ambient temperature $\Theta_{\infty}$, the field $\Theta=\theta \circ \varphi$ indicates the current temperature $\theta(x)$ at a point $x \in \mathscr{B}_{t}$ parametrized by the corresponding material point $\mathbf{X} \in \mathscr{B}_{0}$. Therefore, in the thermoelastic free energy

$$
\Psi^{\text {the }}\left(\tilde{C}_{V}, \tilde{C}_{F}, \Theta\right)=\Psi^{\text {cap }}(\Theta)-2 \sqrt{\tilde{C}_{V}^{2-n_{\text {dim }}}} \beta_{M}\left[\Theta-\Theta_{\infty}\right] \mathrm{D} \Psi_{M}^{\mathrm{vol}}\left(\tilde{C}_{V}\right)-2 \sqrt{\tilde{C}_{F}} \beta_{F}\left[\Theta-\Theta_{\infty}\right] \mathrm{D} \Psi_{F}^{\text {ela }}\left(\tilde{C}_{F}\right)(34)
$$

we take into accout a volume expansion, but also expansion in fiber direction. The heat capacity of matrix and fiber material, respectively, is described by the function $\Psi^{\text {cap }}:=\Psi_{\mathrm{M}}^{\mathrm{cap}}+\Psi_{\mathrm{F}}^{\text {cap }}$ according to the

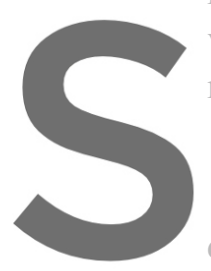
volume fraction of matrix material and fiber bundles
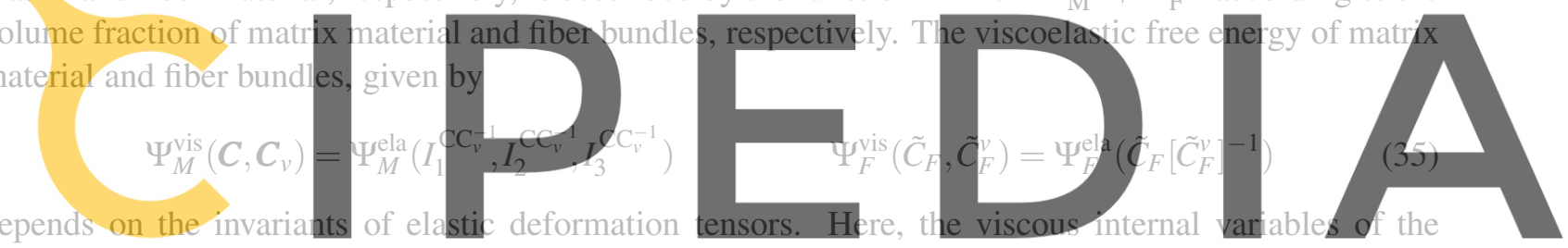

three-dimensional matrix material and the one-dimensional fiber material are designated by $C_{v}$ and $\tilde{C}_{F}^{v}$,

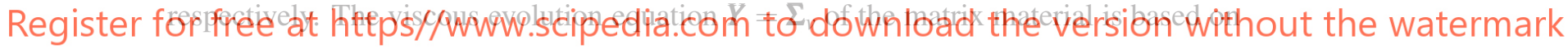

$$
Y:=-\frac{\partial \Psi_{M}^{v i s}}{\partial \mathbf{C}_{v}}
$$

as the non-equilibrium stress tensor, and defines the viscous internal dissipation

$$
D_{M}^{\mathrm{int}}:=\dot{\boldsymbol{C}}_{v}: \boldsymbol{\Sigma}_{v}=\dot{\boldsymbol{C}}_{v}: \mathbb{V}\left(\boldsymbol{C}_{v}\right): \dot{\boldsymbol{C}}_{v} \geq 0
$$

as non-negative-definite quadratic form with respect to the viscosity tensor

$$
\mathbb{V}\left(\boldsymbol{C}_{v}\right)=\frac{1}{4}\left[V_{\mathrm{vol}}-\frac{V_{\mathrm{dev}}}{n_{\mathrm{dim}}}\right] \boldsymbol{C}_{v}^{-1} \otimes \boldsymbol{C}_{v}^{-1}+\frac{V_{\mathrm{dev}}}{4} \boldsymbol{C}_{v}^{-1} \odot \boldsymbol{C}_{v}^{-1} \quad \text { with } \quad \boldsymbol{C}_{v}^{-1} \odot \boldsymbol{C}_{v}^{-1}:=-\frac{\partial \boldsymbol{C}_{v}^{-1}}{\partial \boldsymbol{C}_{v}}
$$

The material parameters $V_{\mathrm{vol}}$ and $V_{\mathrm{dev}}$ designate viscosities with respect to volumetric and deviatoric inelastic deformations of the matrix material. The viscous evolution equation $Y_{F}=\Sigma_{F}^{v}$ of the fiber material is analogously defined by the non-equilibrium stress

$$
Y_{F}:=-\frac{\partial \Psi_{F}^{\mathrm{vis}}}{\partial \tilde{C}_{F}^{v}}
$$


and the viscous internal dissipation

$$
D_{F}^{\mathrm{int}}:=\dot{\tilde{C}}_{F}^{v} \Sigma_{F}^{v}=\dot{\tilde{C}}_{F}^{v} \frac{V_{F}}{4\left(\tilde{C}_{F}^{v}\right)^{2}} \dot{\tilde{C}}_{F}^{v} \geq 0
$$

with the fiber viscosity parameter $V_{F}$.

\section{VARIATIONAL FORMULATION}

The principle of virtual power, in this paper, relies on the total energy balance law of the continuum

$$
\dot{\mathcal{H}}(\underbrace{\dot{\boldsymbol{\varphi}}, \dot{\boldsymbol{v}}, \dot{\boldsymbol{p}}, \dot{\boldsymbol{\alpha}}, \dot{\boldsymbol{\omega}}, \dot{\boldsymbol{\pi}}, \dot{\tilde{\boldsymbol{F}}}, \dot{\tilde{\boldsymbol{G}}}, \dot{\boldsymbol{C}}, \dot{\boldsymbol{C}}_{v}, \dot{\boldsymbol{K}}, \dot{\tilde{C}}_{V}, \dot{\tilde{C}}_{F}, \dot{\tilde{C}}_{F}^{v}, \dot{\Theta}, \dot{\eta}}_{\text {temporally continuous }}, \underbrace{\tilde{\Theta}, \tilde{\boldsymbol{P}}, \boldsymbol{S}, \tilde{\boldsymbol{P}}_{K}, \boldsymbol{S}_{K}, \tilde{\boldsymbol{\tau}}_{\mathrm{skw}}^{t}, \tilde{S}_{V}, \tilde{S}_{F}, \boldsymbol{R}, h, \lambda, \boldsymbol{Z}, \hat{\boldsymbol{\omega}}}_{\text {temporally discontinuous }})=0
$$

where $\mathcal{H}:=\mathcal{T}^{\text {tra }}+\mathcal{T}^{\text {rot }}+\Pi^{\text {ext }}+\Pi^{\text {int }}$. Each field in the argument list has to be temporally independent approximated, but there is a distinction between temporally continuous time rate fields and temporally discontinuous Lagrange multiplier fields. By a tilde symbol in superscript, we highlight a below spatially independent approximated field. A hat symbol in superscript highlights pseudo tensors. In the following, we use a bar symbol in superscript in order to highlight fields, which are parameters of the variational principle, because they are prescribed by the user or the second law of thermodynamics, respectively. In Eq. (41), non-standard fields are especially the material rotation gradient $\tilde{\boldsymbol{G}}$, the curvature-twist tensor $\boldsymbol{K}$, the Piola-Kirchhoff couple stress $\tilde{P}_{K}$ as well as the curvature-twist stress $S_{K}$. In addition to the standard kinetic power functional
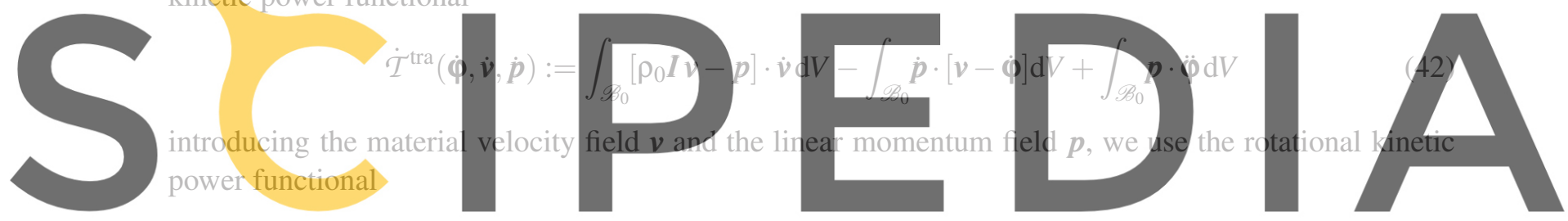

$$
\dot{\mathcal{T}}^{\mathrm{rot}}(\dot{\boldsymbol{\alpha}}, \dot{\omega}, \dot{\pi}):=\int\left[\rho_{0}\left[\left(l_{F}^{2}-l_{0}^{2}\right) A_{0}+l_{0}^{2} \boldsymbol{I}\right] \omega-\pi\right] \cdot \dot{\omega} \mathrm{d} V-\int \dot{\pi} \cdot[\omega-\dot{\alpha}] \mathrm{d} V+\int \pi \cdot \ddot{\alpha} \mathrm{d} V
$$

(43)

Register for free at https//WwW.scipedia.com to download the version without the watermark

for introducing a micro-inertia based on two length scales $l_{F}$ and $l_{0}$. Here, the field $\omega$ denotes the angular

velocity field pertaining to the continuum rotation $\alpha$, and $\pi$ the corresponding angular momentum field.

The external power functional

$$
\begin{aligned}
& \dot{\Pi}^{\text {ext }}:=-\int_{\mathscr{B}_{0}} \rho_{0} \boldsymbol{B} \cdot \dot{\boldsymbol{\varphi}} \mathrm{d} V \quad-\int_{\partial_{T} \mathscr{B}_{0}} \overline{\boldsymbol{T}} \cdot \dot{\boldsymbol{\varphi}} \mathrm{d} A \quad+\int_{\partial_{Q} \mathscr{B}_{0}} \tilde{\Theta} \bar{H} \mathrm{~d} A \\
& +\int_{\mathscr{B}_{0}} \operatorname{Grad}[\tilde{\Theta}] \cdot \boldsymbol{H} \mathrm{d} V+\int_{\mathscr{B}_{0}} \frac{\tilde{\Theta}}{\Theta}\left(D^{\text {cdu }}+D^{\text {int }}\right) \mathrm{d} V+\int_{\mathscr{B}_{0}} \boldsymbol{\Sigma}_{v}: \dot{\boldsymbol{C}}_{v} \mathrm{~d} V \\
& +\int_{\partial_{\Theta} \mathscr{B}_{0}} \lambda\left[\tilde{\Theta}-\Theta_{\infty}\right] \mathrm{d} A-\int_{\partial_{\dot{\Theta}} \mathscr{B}_{0}} h[\dot{\Theta}-\dot{\bar{\Theta}}] \mathrm{d} A-\int_{\partial_{\varphi} \mathscr{B}_{0}} \boldsymbol{R} \cdot[\dot{\boldsymbol{\varphi}}-\dot{\bar{\varphi}}] \mathrm{d} A \\
& -\int_{\partial_{\alpha} \mathscr{B}_{0}} \boldsymbol{Z} \cdot[\dot{\boldsymbol{\alpha}}-\dot{\overline{\boldsymbol{\alpha}}}] \mathrm{d} A-\int_{\partial_{\alpha} \mathscr{B}_{0}} \hat{\boldsymbol{\omega}} \cdot\left[\hat{\boldsymbol{\varepsilon}}: \tilde{\boldsymbol{\tau}}_{\mathrm{skw}}^{t}\right] \mathrm{d} A-\int_{\partial_{W} \mathscr{B}_{0}} \overline{\boldsymbol{W}} \cdot \dot{\boldsymbol{\alpha}} \mathrm{d} A \\
& +\int_{\mathscr{B}_{0}} \frac{1}{2} \bar{S}: \dot{C} \mathrm{~d} V \quad+\int_{\mathscr{B}_{0}} \frac{1}{2} \bar{S}_{V} \dot{\tilde{C}}_{V} \mathrm{~d} V \quad+\int_{\mathscr{B}_{0}} \frac{1}{2} \bar{S}_{F} \dot{\tilde{C}}_{F} \mathrm{~d} V \\
& +\int_{\mathscr{B}_{0}} \Sigma_{F}^{v} \dot{\tilde{C}}_{F}^{v} \mathrm{~d} V \quad+\int_{\mathscr{B}_{0}} \overline{\boldsymbol{S}}_{K}: \dot{\boldsymbol{K}} \mathrm{d} V \quad+\int_{\mathscr{B}_{0}} \bar{M}_{F}^{v} \mathrm{~L}_{\mathrm{F}}\left[\dot{\tilde{C}}_{F}-\dot{\tilde{C}}_{F}^{v}\right] \mathrm{d} V
\end{aligned}
$$


with

$$
\bar{H}:=\frac{\bar{Q}}{\Theta} \quad D^{\mathrm{int}}:=D_{M}^{\mathrm{int}}+D_{F}^{\mathrm{int}} \quad \mathrm{L}_{\mathrm{F}}[\dot{\bullet}]=\frac{\overline{\ln (\bullet)}}{2}
$$

includes algorithmic stresses $\bar{S}, \bar{S}_{V}, \bar{S}_{F}, \bar{S}_{K}$ and $\bar{M}_{F}^{v}$ in order to obtain an energy-momentum scheme, a mass specific volume load $\boldsymbol{B}$ and traction loads $\overline{\boldsymbol{T}}$ on the Neumann boundary $\partial_{T} \mathscr{B}_{0}$, thermal loads $\bar{Q}$ on the thermal Neumann boundary $\partial_{Q} \mathscr{B}_{0}$, the Fourier heat conduction

$$
\boldsymbol{H}:=-\frac{1}{\Theta}\left[\frac{k_{\mathrm{F}_{0}}-k_{0}}{\tilde{C}_{\mathrm{F}}} \boldsymbol{A}_{0}+k_{0}\left[\tilde{\boldsymbol{F}}^{t} \tilde{\boldsymbol{F}}\right]^{-1}\right] \operatorname{Grad}[\Theta] \quad \text { with } \quad D^{\text {cdu }}:=-\operatorname{Grad}[\Theta] \cdot \boldsymbol{H} \geq 0
$$

as corresponding dissipation, the viscous time evolutions, Dirichlet boundaries $\partial_{\Theta} \mathscr{B}_{0} \cup \partial_{\Theta} \mathscr{B}_{0}:=\partial \mathscr{B}_{0} \backslash$ $\partial_{Q} \mathscr{B}_{0}$ with $\partial_{\Theta} \mathscr{B}_{0} \cap \partial_{\Theta} \mathscr{B}_{0}=\emptyset$ for prescribing the ambient temperature $\Theta_{\infty}$ or the time evolution $\bar{\Theta}$ of the temperature, respectively, as well as prescribed displacements $\bar{\varphi}-\boldsymbol{X}$ on $\partial_{\varphi} \mathscr{B}_{0}$. This constrained micropolar continuum also allows for a Neumann boundary $\partial_{W} \mathscr{B}_{0}$ for prescribing boundary couples $\bar{W}$, and a Dirichlet rotation boundary $\partial_{\alpha} \mathscr{B}_{0}:=\partial \mathscr{B}_{0} \backslash \partial_{W} \mathscr{B}_{0}$, which allows for boundary rotations $\bar{\alpha}$. Since the Dirichlet boundaries have to be formally introduced by Lagrange multiplier terms, $\lambda$ denotes a boundary entropy flux, $h$ a boundary entropy and $\boldsymbol{R}$ Newtonian boundary forces. The Lagrange multiplier field $\mathrm{Z}$ includes the reaction couples on the boundary $\partial_{\alpha} \mathscr{B}_{0}$, and $\hat{\boldsymbol{\omega}}$ the reaction angular velocities arising from the orthogonality property with the skew-symmetric Kirchhoff stress $\tilde{\tau}_{\mathrm{skw}}^{t}$. Here, we applied the third-order permutation tensor $\hat{\varepsilon}$. The skew-symmetric Kirchhoff stress $\tilde{\tau}_{\text {skw }}^{t}$ is a Lagrange multiplier
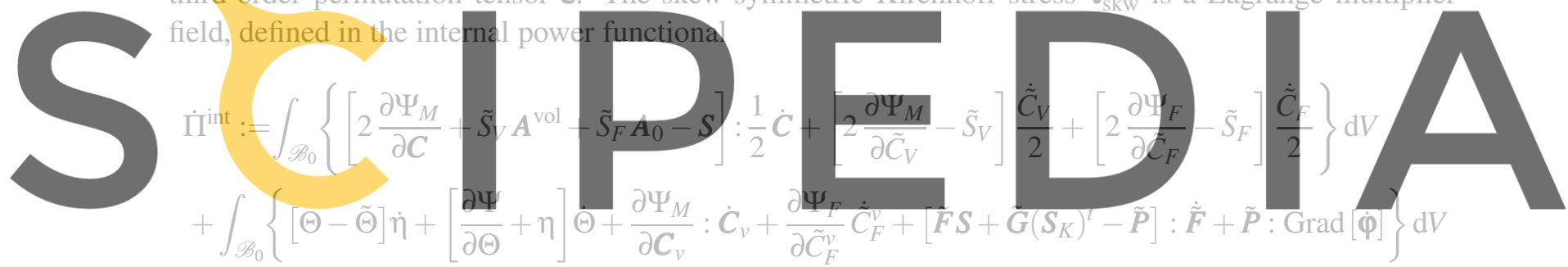

Register for free at https//www.scipedia.com to download the version without the watermark $+\int_{\mathscr{B}_{0}}\left\{\tilde{\mathbb{P}}_{K}: \operatorname{Grad}[\dot{\alpha}]+\tilde{\tau}_{\text {skw }}^{t}: \hat{\varepsilon} \cdot\left[\frac{1}{2} \hat{\varepsilon}: \dot{\tilde{F}} \tilde{F}^{-1}+\dot{\alpha}\right]+\left[\tilde{F} S_{K}-\tilde{P}_{K}\right]: \dot{\tilde{G}}+\left[\frac{\partial \Psi_{K}}{\partial K}-S_{K}\right]: \dot{K}\right\} \mathrm{d} V$

with

$$
\boldsymbol{A}^{\mathrm{vol}}(\boldsymbol{C}):=\frac{1}{n_{\mathrm{dim}}} \operatorname{det}(\boldsymbol{C})^{\frac{1}{n_{\mathrm{dim}}}} \boldsymbol{C}^{-1} \quad \Psi_{\mathrm{M}}:=\Psi_{\mathrm{M}}^{\mathrm{iso}}+\Psi_{\mathrm{M}}^{\mathrm{vol}}+\Psi_{\mathrm{M}}^{\mathrm{vis}}+\Psi_{\mathrm{M}}^{\text {the }} \quad \Psi_{\mathrm{F}}:=\Psi_{\mathrm{F}}^{\text {ela }}+\Psi_{\mathrm{F}}^{\mathrm{vis}}+\Psi_{\mathrm{F}}^{\text {the }}
$$

in order to introduce the strain power arising from the drilling degrees of freedom. The free energy $\Psi:=$ $\Psi_{\mathrm{M}}+\Psi_{\mathrm{F}}$ indicates the total free energy of the composite. The assumed temperature field $\tilde{\Theta}$ replaces a thermal displacement field, being not explicitly involved in the total free energy according to the assumed Fourier heat conduction, and $\eta$ denotes the entropy density of the composite. After varying the total energy balance law in Eq. (41) with respect to temporally continuous time rate fields and temporally discontinuous Lagrange multipliers, we apply the fundamental theorem of calculus of variations in

$$
\delta_{*} \dot{\mathcal{H}}(\underbrace{\dot{\boldsymbol{\varphi}}, \dot{\boldsymbol{v}}, \dot{\boldsymbol{p}}, \dot{\boldsymbol{\alpha}}, \dot{\boldsymbol{\omega}}, \dot{\boldsymbol{\pi}}, \dot{\tilde{\boldsymbol{F}}}, \dot{\tilde{\boldsymbol{G}}}, \dot{\boldsymbol{C}}, \dot{\boldsymbol{C}}_{v}, \dot{\boldsymbol{K}}, \dot{\tilde{C}}_{V}, \dot{\tilde{C}}_{F}, \dot{\tilde{C}}_{F}^{v}, \dot{\Theta}, \dot{\eta}}_{\text {temporally continuous }}, \underbrace{\tilde{\Theta}, \tilde{\boldsymbol{P}}, \boldsymbol{S}, \tilde{\boldsymbol{P}}_{K}, \boldsymbol{S}_{K}, \tilde{\boldsymbol{\tau}}_{\mathrm{skw}}^{t}, \tilde{S}_{V}, \tilde{S}_{F}, \boldsymbol{R}, h, \lambda, \boldsymbol{Z}, \hat{\boldsymbol{\omega}}}_{\text {temporally discontinuous }})=0
$$


and arrive at non-standard weak forms on the volume of the constrained micropolar continuum, given by

$$
\begin{aligned}
& \int_{\mathscr{B}_{0}} \boldsymbol{\delta}_{*} \dot{\boldsymbol{K}}:\left[\frac{\partial \Psi_{K}}{\partial \boldsymbol{K}}+\overline{\boldsymbol{S}}_{K}-\boldsymbol{S}_{K}\right] \mathrm{d} V=0=\int_{\mathscr{B}_{0}} \boldsymbol{\delta}_{*} \dot{\tilde{\boldsymbol{G}}}:\left[\tilde{\boldsymbol{F}} \boldsymbol{S}_{K}-\tilde{\boldsymbol{P}}_{K}\right] \mathrm{d} V \\
& \int_{\mathscr{B}_{0}} \boldsymbol{\delta}_{*} \tilde{\boldsymbol{P}}_{K}:[\operatorname{Grad}[\dot{\boldsymbol{\alpha}}]-\dot{\tilde{\boldsymbol{G}}}] \mathrm{d} V=0=\int_{\mathscr{B}_{0}} \boldsymbol{\delta}_{*} \dot{\boldsymbol{\pi}} \cdot[\dot{\boldsymbol{\alpha}}-\boldsymbol{\omega}] \mathrm{d} V \\
& \int_{\mathscr{B}_{0}} \boldsymbol{\delta}_{*} \dot{\boldsymbol{\omega}} \cdot\left[\rho_{0}\left[\left(l_{F}^{2}-l_{0}^{2}\right) \boldsymbol{A}_{0}+l_{0}^{2} \boldsymbol{I}\right] \boldsymbol{\omega}-\boldsymbol{\pi}\right] \mathrm{d} V=0=\int_{\mathscr{B}_{0}} \boldsymbol{\delta}_{*} \boldsymbol{S}_{K}:\left[\dot{\tilde{\boldsymbol{F}}}^{t} \tilde{\boldsymbol{G}}+\tilde{\boldsymbol{F}}^{t} \dot{\tilde{\boldsymbol{G}}}-\dot{\boldsymbol{K}}\right] \mathrm{d} V \\
& \int_{\mathscr{B}_{0}} \boldsymbol{\delta}_{*} \dot{\boldsymbol{\alpha}} \cdot\left[\dot{\boldsymbol{\pi}}+\hat{\boldsymbol{\varepsilon}}: \tilde{\boldsymbol{\tau}}_{\mathrm{skw}}^{t}\right] \mathrm{d} V+\int_{\mathscr{B}_{0}} \tilde{\boldsymbol{P}}_{K}: \operatorname{Grad}\left[\boldsymbol{\delta}_{*} \dot{\boldsymbol{\alpha}}\right] \mathrm{d} V=\int_{\partial_{\alpha} \mathscr{B}_{0}} \boldsymbol{\delta}_{*} \dot{\boldsymbol{\alpha}} \cdot \boldsymbol{Z} \mathrm{d} A+\int_{\partial_{W} \mathscr{B}_{0}} \boldsymbol{\delta}_{*} \dot{\boldsymbol{\alpha}} \cdot \overline{\boldsymbol{W}} \mathrm{d} A \\
& \int_{\mathscr{B}_{0}} \delta_{\alpha} \tilde{\tau}_{\mathrm{skw}}^{t}: \hat{\boldsymbol{\varepsilon}} \cdot\left[\frac{1}{2} \hat{\boldsymbol{\varepsilon}}: \dot{\tilde{\boldsymbol{F}}} \tilde{\boldsymbol{F}}^{-1}+\dot{\boldsymbol{\alpha}}\right] \mathrm{d} V=\int_{\partial_{\alpha} \mathscr{B}_{0}} \hat{\boldsymbol{\omega}} \cdot \hat{\boldsymbol{\varepsilon}}: \delta_{*} \tilde{\tau}_{\mathrm{skw}}^{t} \mathrm{~d} A
\end{aligned}
$$

In Eqs. (50), we obtain the weak forms corresponding to the curvature-twist stress tensor $\boldsymbol{S}_{K}$ and the Piola-Kirchhoff couple stress tensor $\tilde{\boldsymbol{P}}_{K}$, respectively. Eqs. (51) show the weak forms of the material rotation gradient $\tilde{G}$ and the rotational velocity $\boldsymbol{\omega}$. The weak form of the rotational momentum and the curvature-twist tensor are given in Eqs. (52). In Eq. (53), we show the balance of spin angular momentum for determining $\tilde{\tau}_{\text {skw }}^{t}$, where Eq. (54) includes the weak form defining the continuum rotation vector or drilling degrees of freedom, respectively, $\alpha$. The remainder weak forms and the algorithmic stresses $\bar{S}$, $\bar{S}_{V}, \bar{S}_{F}$ and $\bar{M}_{F}^{v}$ are presented in Reference [10], and references therein.

The curvature-twist strain energy $\Psi_{K}$ also leads to a new contribution in the balance law of potential energy on a time step, g
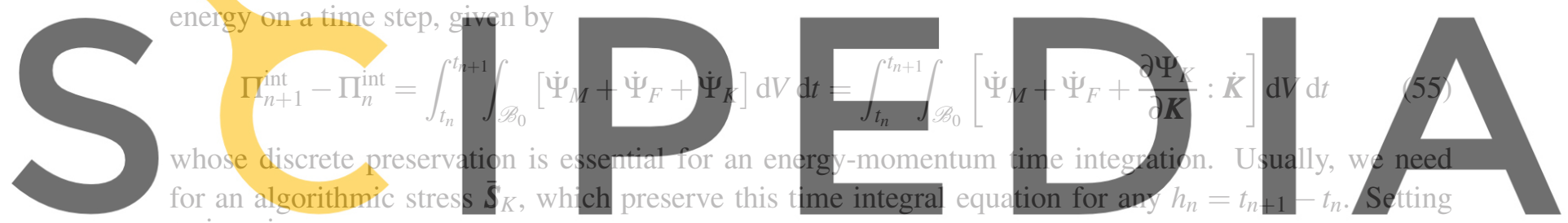

$\delta_{*} \dot{K}=\dot{K}$ and $\delta_{*} S_{K}=S_{K}$, we obtain the weak forms

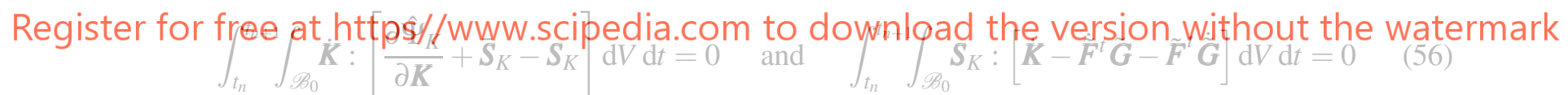

and arrive by employing Eq. (55) at the integral equations

$$
\begin{aligned}
\int_{t_{n}}^{t_{n+1}} \int_{\mathscr{B}_{0}} \overline{\boldsymbol{S}}_{K}: \dot{\boldsymbol{K}} \mathrm{d} V \mathrm{~d} t & =\Pi_{n+1}^{\mathrm{int}}-\Pi_{n}^{\mathrm{int}}-\int_{t_{n}}^{t_{n+1}} \int_{\mathscr{B}_{0}}\left\{\dot{\Psi}_{M}+\dot{\Psi}_{F}+\boldsymbol{S}_{K}: \dot{\boldsymbol{K}}\right\} \mathrm{d} V \mathrm{~d} t \\
& =\Pi_{n+1}^{\mathrm{int}}-\Pi_{n}^{\mathrm{int}}-\int_{t_{n}}^{t_{n+1}} \int_{\mathscr{B}_{0}}\left\{\dot{\Psi}_{M}+\dot{\Psi}_{F}+\boldsymbol{S}_{K}:\left[\dot{\tilde{\boldsymbol{F}}}^{t} \tilde{\boldsymbol{G}}+\tilde{\boldsymbol{F}}^{t} \dot{\tilde{\boldsymbol{G}}}\right]\right\} \mathrm{d} V \mathrm{~d} t
\end{aligned}
$$

In this way, a constraint for the algorithmic stress $\overline{\boldsymbol{S}}_{K}$ for any time step size $h_{n}$ can be written as (cf. [11])

$$
\Psi_{K_{n+1}}-\Psi_{K_{n}}-\int_{0}^{1} \boldsymbol{S}_{K}: \frac{\partial \boldsymbol{K}}{\partial \alpha} \mathrm{d} \alpha=\int_{0}^{1} \overline{\boldsymbol{S}}_{K}: \frac{\partial \boldsymbol{K}}{\partial \alpha} \mathrm{d} \alpha \quad \alpha(t):=\frac{t-t_{n}}{h_{n}}
$$

But, by approximating in Eq. (58) the curvature-twist tensor $\boldsymbol{K}$ and the time integral such, that

$$
\boldsymbol{K}(\alpha):=\sum_{I=1}^{k+1} M_{I}(\alpha) \boldsymbol{K}^{I} \quad M_{I}(\alpha):=\prod_{\substack{J=1 \\ I \neq J}}^{k+1} \frac{\alpha-\alpha_{J}}{\alpha_{I}-\alpha_{J}} \quad I_{\text {Gauss }}\{f\}=\sum_{l=1}^{k} f\left(\xi_{l}\right) w_{l}
$$



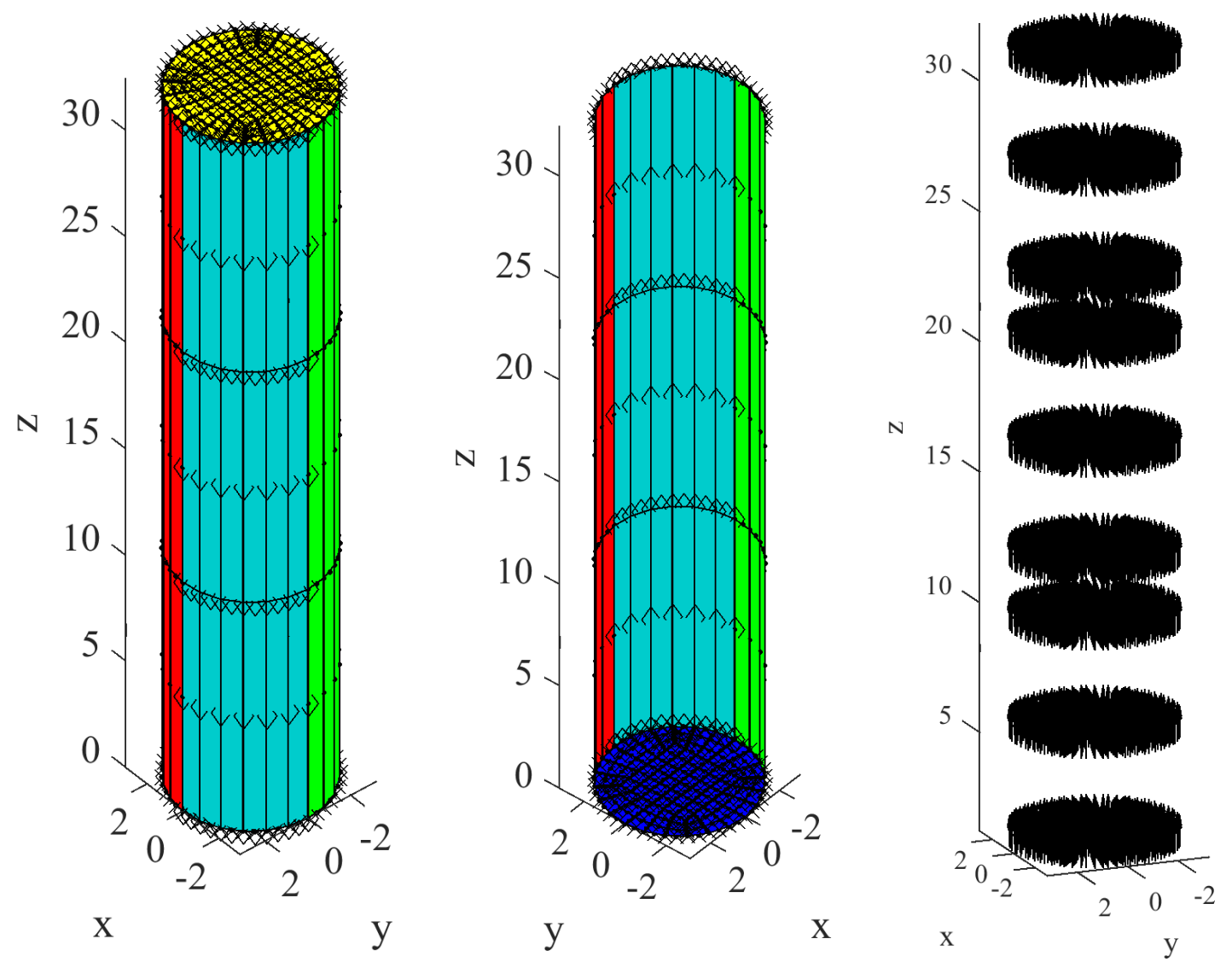

Figure 3: Boundary conditions and fiber bundles indicated by colors, markers and black arrows, respectively.

with Lagrange polynomials $M_{I}(\alpha), I=1, \ldots, k+1$, in time, a Gaussian quadrature rule $I_{\text {Gauss }}$ with Gauss points $\xi_{l}$ and weights $w_{l}, l=1, \ldots, k$, Eq. (58) is satisfied for St. Venant-Kirchhoff material. Therefore, an algorithmic curvature-twist stress $\overline{\boldsymbol{S}}_{K}$ vanishes in this case, which is well-known from a St. VenantKirchhoff matrix material (cf. Reference [8]).

\section{NUMERICAL EXAMPLE}

We demonstrate by numerical examples the behaviour of the St. Venant-Kirchhoff fiber curvature-twist stress. We consider Taylor's bar with fiber bundels along the bar axis (see Figure 3). First, we consider a dynamic torsion by using an axial transient couple load $W_{z}(\boldsymbol{X})=\mp W_{z}\left[f_{L} \sin \omega_{L} t\right]^{2}$ on each point $\boldsymbol{X} \in \partial_{W} \mathscr{B}_{0}$. On the yellow bar end, we prescribe the time evolution of the torque in $-z$-direction, and on the blue end in $z$-direction. Both ends, are also a thermal Dirichlet boundary $\partial_{\Theta} \mathscr{B}_{0}$ with $\Theta(\boldsymbol{X})=\Theta_{\infty}$. The green, red and light blue lateral surfaces are thermally and mechanically insulated in these examples. As usual for Taylor's bar, we also prescribe an initial velocity field $\boldsymbol{v}(\boldsymbol{X})=-v_{0} \boldsymbol{e}_{z}$. The time integration is performed by the 121-em energy-momentum scheme with linear finite elements in time for mechanical and viscous quantities, and quadratic finite elements in time for thermal quantities. The space approximation follows from Reference [12]. Then, we consider a dynamic bending by using a transient couple load $W_{y}(\boldsymbol{X})=\mp W_{y}\left[f_{L} \sin \omega_{L} t\right]^{2}$, where $\omega_{L}$ denotes the excitation frequency, and $f_{L}$ a load cycle weight. 


\section{CONCLUSIONS}

Motivated by precise simulations of fiber roving composites with fiber twisting and bending stiffness on the micro scale, we aim at energy-momentum schemes for constrained micropolar continua, derived by a principle of virtual power for continua. Therefore, we introduce independent fields for the continuum rotation, and discretize by using new mixed finite elements. Important results are that a torsional and flexural rigidity of fibers can be prescribed, even separately. Next design steps are a nonlinear curvaturetwist strain energy function and the corresponding algorithmic curvature-twist stress tensor.

\section{REFERENCES}

[1] De Luycker, E. and Morestin, F. and Boisse, P. and Marsal, D. Simulation of 3D interlock composite preforming. Composite Structures (2009) 88(4):615-623.

[2] Steigmann, D. J. and Dell' Isola, F. Mechanical response of fabric sheets to three-dimensional bending, twisting, and stretching. Acta Mechanica Sinica (2015) 31:373-382.

[3] Hughes, T.J.R and Brezzi, F. On drilling degrees of freedom. Comput. Methods Appl. Mech. Engrg. (1989) 72(1):105-121.

[4] Steinmann, P. and Stein, E. A unifying treatise of variational principles for two types of micropolar continua. Acta Mechanica (1997) 121:215-232.

[5] Madeo, A. and Ferretti, M. and Dell'Isola, F. and Boisse, P. Thick fibrous composite reinforcements behave as special second-gradient materials: three-point bending of 3D interlocks. Zeitschrift für angewandte Mathematik und Physik (2015) 66(4):2041-2060.

[6] Stokes, V.K. Theories of fluids with microstructure: An introduction, Springer Science \& Business Media (2012).

[7] Marsden, J. E. and Hughes, Thomas J. R. Mathematical foundations of elasticity, Englewood Cliffs, N.J., Prentice-Hall (1983).

[8] Simo, J.C. and Tarnow, N. The Discrete Energy-Momentum Method. Conserving Algorithms for Nonlinear Elastodynamics. Z. angew. Math. Phys. (1992) 43:757-792.

[9] Grbčić, S. and Ibrahimbegović, A. and Jelenić, G. Variational formulation of micropolar elasticity using 3D hexahedral finite-element interpolation with incompatible modes. Computers \& Structures (2018) 205:1-14.

[10] Groß, M., Dietzsch J., Röbiger, C. Non-isothermal energy-momentum time integrations with drilling degrees of freedom of composites with viscoelastic fiber bundles and curvature-twist stiffness. Comput. Methods Appl. Mech. Engrg. (2020) 365, in print, doi.org/10.1016/j.cma.2020.112973.

[11] Groß, M. and Betsch, P. and Steinmann, P. Conservation properties of a time FE method. Part IV: higher order energy and momentum conserving schemes. Int. J. Numer. Methods Engng. (2005) 63:1849-1897.

[12] Groß, M. and Dietzsch, J. and Röbiger, C. A mixed B-bar formulation derived by a principle of virtual power for energy-momentum time integrations of fiber-reinforced continua. Comput. Methods Appl. Mech. Engrg. (2019) 350:595-640. 